\title{
Application Strategy of BIM Technology in Highway and Bridge Design
}

\section{Zhongyu Wang*}

China Merchants Chongqing Communications Research \& Design Institute Co., Ltd., Chongqing 400067, China

\begin{abstract}
For the highway and bridge construction industry, the traditional management model has been difficult to adapt to the current information management background. In recent years, in order to comprehensively improve the information management level of highway and bridge engineering, the highway and bridge industry advocates the use of emerging technologies to achieve full-cycle dynamic management of highway and bridge engineering management, and try to ensure the quality of highway and bridge engineering management while improving The efficiency of highway and bridge design management. In view of this, this article is mainly based on the background of information management, researching and analyzing the application strategy of BIM technology in highway bridge design for reference.
\end{abstract}

Key words: BIM technology; Highway bridge design; Application strategy

Publication date: May, 2021; Publication online: 31 May, 2021

*Corresponding author: Zhongyu Wang, ccrdiyu@gmail.com

\section{Analysis of the application advantages of BIM}

\section{technology}

\subsection{D visualization function}

The BIM technology embodies the core technical content mainly through the use of computer terminals or the Internet to construct a scientific and reasonable three-dimensional model of an engineering project. And use digital technology to realize the integrity and real-time analysis of the three-dimensional model of construction engineering. For example, the geometric information and physical properties of building components can be obtained through analysis, and key information such as non-component objects such as space and motion behavior can also be obtained through analysis $^{[1]}$.

It is not difficult to see that through the rational use of the three-dimensional visual information model, the degree of data integration of the project has been significantly strengthened, providing a good platform for information exchange and information sharing for relevant stakeholders. In addition, decision makers can also use the virtual construction method in the BIM $3 \mathrm{D}$ visualization function to merge structural parts to form a 3D solid structure. And in accordance with the 1:1 simulation form, the three-dimensional entity structure is drawn and analyzed, and the project structure model is output in the form of drawings.

\subsection{Digital application characteristics}

BIM technology is a derivative product of the development of modern computer technology, and has the characteristics of informatization and digitization in terms of technical application characteristics. Among them, in terms of the performance of the characteristics of digital applications, research and analysis can be conducted mainly from three aspects: completeness, relevance and consistency. Taking completeness as an example, all the information in the model needs to include $3 \mathrm{D}$ geometric information, engineering information, and so on. In terms of relevance, if an object in the model changes, the corresponding prefabricated information will also change significantly. In addition, in terms of consistency, the same information only needs to be entered once in the entire life cycle to achieve the entire process control effect ${ }^{[2]}$.

\subsection{Advantages of collaborative management}


The collaborative management advantages of BIM technology can help all parties involved in a project to jointly solve problems and ensure that information transparency is strengthened. For example, in the design stage, collaborative design can be carried out between different disciplines. Different majors can coordinate and deal with the contradictions in the current design work according to the information feedback, so as to reduce the contradictions and risk problems that exist during the subsequent construction operations. For example, the main body of the project can take advantage of the collaborative management of BIM technology to conduct a comprehensive simulation analysis of the safety and economy of the project. According to the analysis and feedback results, the control and management efforts were strengthened from the aspects of design, construction and operation and maintenance management.

\section{Analysis of application strategy of BIM}

\section{technology in highway bridge design}

\subsection{Construction strategy of collaborative design platform based on BIM technology}

At present, bridge professional designers have actively combined the advantages of BIM technology to fully develop, promote and apply BIM design software. In the specific application process, the design basis is mainly based on the results of digital survey and design. Combine the content of the design data to construct a scientific and reasonable BIM model of the bridge plan. And according to the functional advantages of collaborative design, the BIM model of each professional information and the environmental model are integrated and applied to ensure that the design effect of the large-scenario BIM model can meet the expectations ${ }^{[3]}$.

In this process, professional designers need to actively combine professional characteristics and technical standards to accurately judge the feasibility of the current bridge design plan. It should be noted that all majors must ensure that the design schemes are properly connected in order to achieve collaborative processing effects. Or you can also use the BIM collaboration platform, after review and confirmation by various professional designers, appropriate improvements and adjustments to the relevant design content to ensure that the information transmission function and application function can meet expectations.

\subsection{Application strategies selected for comparison of bridge schemes}

The prefabricated bridges account for a large proportion of the entire line of bridges, and traditional bridge design methods are difficult to achieve optimization, improvement and dynamic management of the overall design of prefabricated bridges. Although from an objective point of view, traditional highway bridge design is mainly based on 1:2000 topographic maps and route design data for planning and design, and can achieve the goal of rapid design, but this design method is prone to imperfect structural structure and the location of the crossed object Problems with unclear relationships.

If it is applied to on-site construction operations, it is prone to construction risks. Through the scientific use of BIM technology, the staff can construct the highway bridge BIM model in advance. Utilize the visualization function and collaborative design function of BIM technology to identify and prevent potential risks in the construction site in advance. And with the aid of the BIM model, the bridge construction content is collaboratively designed to solve the problem of imperfect structural structure or unclear positional relationship of the crossed objects in the past ${ }^{[4]}$.

\subsection{Application strategy in steel bridge detail design}

Based on previous construction design experience, the more common bridge types are mainly long-span rigid frame bridges and cable-stayed bridges. Due to the complexity of this part of the bridge itself, complex node methods should be adopted for targeted treatment in the design process. In other words, the traditional two-dimensional drawing method has been difficult to meet the current bridge design requirements. It is suggested that the designer can use BIM technology to make overall planning and reasonable deployment of the detailed design content of the steel bridge.

On the one hand, the visualization function and simulation function of BIM technology can be applied to the structural design of steel truss main girder node anchor bolts and cable saddles. According to the data analysis and feedback, the designer can predict the error problems that may occur in the current on-site construction in advance, and reduce the occurrence of hidden construction hazards.

\subsection{Application strategies in the disclosure of bridge visualization technology}

Scientific use of the visualization function of BIM technology can efficiently complete the technical 
clarification work. For example, designers can use the simulation function of BIM technology to realize the simulation analysis of each key construction process. According to the analysis and feedback results, the design intention is directly expressed, and the key points are marked during the construction operation stage to ensure the efficiency of on-site communication and the quality of work to be deepened and strengthened.

\section{Conclusion}

All in all, the promotion and application of BIM technology has effectively overcome the disadvantages of traditional highway bridge design to a certain extent, such as breaking through the previous design mode dominated by human experience, and not only effectively improving the safety and reliability of the design process At the same time, it also promotes the realization of interconnection between people, visual information models and structures to ensure the efficient operation of highway and bridge projects. However, it should be noted that the current BIM technology in my country has not yet reached the stage of mature application, and there are still problems that need to be overcome in some technical systems. Therefore, in the future development, relevant researchers should strengthen the research on BIM technology to ensure that the highway bridge design system based on BIM technology can be more intuitive and integrated, and provide a good guarantee for the sustainable development of my country's bridge construction industry.

\section{Disclosure statement}

The author declares no conflict of interest.

\section{References}

[1] Yue LF. Discussion on the design of highway bridge based on BIM[J]. China Construction, 2019(03):166-167.

[2] Ma B. Analysis of the application of BIM technology in highway bridge design[J]. Sichuan Cement, 2019(08): 54.

[3] Wang J. The application of BIM technology in highway bridge design $[\mathrm{J}]$. Heilongjiang Transportation Science and Technology, 2021, 44(01): 246-247.

[4] Wang LW, Ren Jiao. Application of BIM technology in highway bridge design [J]. Heilongjiang Transportation Science and Technology, 2021, 44(03): 236-237. 